\title{
New aspects on chromosomal instability: Chromosomal break-points in Fanconi anemia patients co-localize on the molecular level with fragile sites
}

\author{
CHRISTIANE SCHODER ${ }^{1}$, THOMAS LIEHR ${ }^{1}$, EUNIKE VELLEUER ${ }^{2}$, KATHLEEN WILHELM $^{1}$, \\ NANCY BLAUROCK ${ }^{1}$, ANJA WEISE ${ }^{1}$ and KRISTIN MRASEK ${ }^{1}$ \\ ${ }^{1}$ Jena University Hospital, Institute of Human Genetics and Anthropology, Kollegiengasse 10, \\ D-07743 Jena; ${ }^{2}$ Klinik für Kinder-Onkologie, -Hämatologie und Klinische Immunologie, \\ Universitäts-klinikum Düsseldorf, Moorenstrasse 5, D-40225 Düsseldorf, Germany
}

Received September 15, 2009; Accepted October 30, 2009

DOI: 10.3892/ijo_00000501

\begin{abstract}
Within cytogenetic preparations chromosomal breaks can be observed in patients suffering from Fanconi anemia (FA), a recessively inherited syndrome with an extremely elevated cancer risk, but also in healthy individuals as so-called fragile sites (FS). It is known that FS cytogenetically co-localize with tumor- and evolutionaryconserved chromosomal break-points. The also suggested colocalization of FS and FA associated break-points (FA-bp) was studied here for the first time systematically by molecular cytogenetics. Metaphase chromosomes were obtained from lymphocytes of two FA patients (FANC-A and FANC-C, respectively). Overall $50.58 \%$ of the investigated FA-bp correspond to cytogenetic regions with known FS. A detailed molecular cytogenetic study applying FS-spanning probes revealed that 24/29 (82.8\%) of analyzed FS are in concordance with FA-bp. Notably, FA-bp show a distribution pattern deviating from that of Aphidicolin induced FS. FA-bp appear more frequently within GTG-light bands and additionally, a yet unreported correlation was observed between break rate and chromosomal banding level. In future, FA-bp might serve as model for the mapping and analysis of otherwise rarely observable FS.
\end{abstract}

\section{Introduction}

Fanconi anemia (FA) is a so called 'chromosomal breakage syndrome' inherited in an autosomal or X-chromosomal recessive manner with a prevalence estimated at 1:100,000 live births (1). Cells of FA-patients show hypersensitivity to

Correspondence to: Dr Anja Weise, Institut für Humangenetik, Postfach, D-07740 Jena, Germany

E-mail: aweise@mti.uni-jena.de

Key words: Fanconi anemia, Fragile sites, chromosome instability
DNA-crosslinking agents such as diepoxybutane, cisplatin and mitomycin C (MMC) (2). Cytogenetically, FA is characterized by a high incidence of chromosomal gaps, breaks and rearrangement figures consisting of several chromosomes or chromosome parts. FA is caused by different defects in proteins of the pathway responsible for DNA double strand breakage repair. Currently 13 complementation groups are reported; $90 \%$ of them constitute FANC-A, -C, -G, -D1 and -D2 (3). Seventy-five percent of FA-patients show constitutional abnormalities such as deformities of thumb and radius, heart, urogenital and gastrointestinal tract, microcephaly, microphthalmy and microsomy. Progressive bone marrow failure and a resulting anemia are the characteristic symptoms of FA together with a high incidence of leukemia and early onset of (squamous cell) cancer. The latter are thought to be linked to the high rate of chromosome breakage. In this context, FA seems to be an interesting model for carcinogenesis not only on the moleculargenetic level (4) but also on the chromosomal level.

Chromosomal breaks do not only occur in specific syndromes and cancer they are also part of the normal chromosome structure and can be induced by specific culture conditions. These so-called fragile sites (FS) are chromosomal regions that show a high incidence of gaps and breaks in metaphase chromosomes of healthy individuals. They are thought to be expressed due to partial inhibition of DNA synthesis and represent sites of late replication (5). The expression of FS can even be augmented by adding aphidicolin, an inhibitor of polymerase $\alpha$ (6). Although the reason for their fragility is not completely been elucidated yet, all FS examined so far show AT-rich islands and an elevated incidence of DNA areas with high flexibility (7). It was demonstrated that the lack of special proteins like ATR leads to a higher FS expression and are therefore involved in the maintenance of chromosome stability (8).

FS are involved in chromosomal rearrangements (9), virus integration (10), sister-chromatide exchange (11) and gene amplification (12). Because of these features FS can contribute to oncogenesis (13). Furthermore, several FS include tumor suppressor genes such as the FHIT-gene in FRA3B or the WWOX-gene in FRA16D (14). Due to these findings FS 
Table I. Summary of the number of FS-specific BAC-probes applied for FISH to test co-localization with FA-bp, and results obtained. ${ }^{\mathrm{a}}$

\begin{tabular}{|c|c|c|c|}
\hline $\begin{array}{l}\text { No. of FS } \\
\text { studied }\end{array}$ & $\begin{array}{c}\text { Fragile site/chromosomal } \\
\text { localization }\end{array}$ & $\begin{array}{l}\text { BAC probe specific } \\
\text { for fragile site }\end{array}$ & $\begin{array}{l}\text { Co-localization of } \\
\text { FS and FA-bp }\end{array}$ \\
\hline 1 & FRA1D/1p22 & RP11-145D11 & + \\
\hline 2 & FRA1A/1p36 & RP11-19M4 & + \\
\hline 3 & FRA1F/1q21 & RP11-301M17 & + \\
\hline \multirow[t]{2}{*}{4} & FRA1K/1q31 & RP11-258M18 & + \\
\hline & & RP11-417A21 & + \\
\hline 5 & FRA2L/2p11 & RP11-433C18 & + \\
\hline 6 & FRA2F/2q21.3 & RP11-442L5 & + \\
\hline 7 & FRA2G/2q31 & RP11-234L7 & + \\
\hline 8 & FRA2H/2q32 & RP11-372A6 & + \\
\hline 9 & FRA2J/2q37.3 & RP11-1345D17 & + \\
\hline 10 & FRA4C/4q31.1 & RP11-364L4 & + \\
\hline \multirow[t]{2}{*}{11} & FRA6I/6p11 & RP11-91N13 & - \\
\hline & & RP11-452D24 & + \\
\hline \multirow[t]{2}{*}{12} & FRA6G/6q15 & RP11-482L14 & + \\
\hline & & RP11-524K14 & + \\
\hline \multirow[t]{2}{*}{13} & FRA6F/6q21 & RP11-71B11 & + \\
\hline & & RP11-188A15 & + \\
\hline 14 & FRA6E/6q26 & RP11-421L20 & + \\
\hline 15 & FRA7C/7p14.4 & RP11-552B14 & + \\
\hline 16 & FRA7B/7p22 & RP11-172O13 & - \\
\hline 17 & FRA7J/7q11 & RP11-219M8 & + \\
\hline 18 & FRA7E/7q21.2 & RP11-575G1 & + \\
\hline \multirow[t]{2}{*}{19} & FRA7F/7q22 & RP11-269I22 & + \\
\hline & & RP11-605O24 & + \\
\hline \multirow[t]{2}{*}{20} & FRA8C/8q24.1 & RP11-468O2 & + \\
\hline & & RP11-414A9 & + \\
\hline 21 & FRA10G/10q11.2 & RP11-556L1 & + \\
\hline 22 & FRA11F/11q14.2 & RP11-529A4 & + \\
\hline \multirow[t]{2}{*}{23} & FRA11G/11q23.3 & RP11-433N6 & - \\
\hline & & RP11-67G8 & + \\
\hline \multirow[t]{2}{*}{24} & FRA13A/13q13.2 & RP11-66B6 & + \\
\hline & & RP11-98D3 & - \\
\hline 25 & FRA13B/13q21.2 & RP11-129M14 & + \\
\hline \multirow[t]{2}{*}{26} & FRA13E/13q22 & RP11-132L12 & + \\
\hline & & RP11-555G22 & + \\
\hline 27 & FRA14C/14q24.1 & RP11-179B8 & - \\
\hline 28 & FRA16C/16q22.1 & RP11-157E19 & + \\
\hline 29 & FRA16D/16q23.2 & RP11-358L22 & + \\
\hline
\end{tabular}

${ }^{\mathrm{a}} \mathrm{FS}$ and chromosomal localization are listed according to Lukusa and Fryns (22).

became a novel significance in the field of cancer genetics. Besides cancer, FS play a role in chromosome evolution in hominoidae: there is evidence that evolutionary conserved break-points co-localize with known FS on the molecularcytogenetic level (15). Therefore, FS seem to act as 'hot-spots of recombination'. 


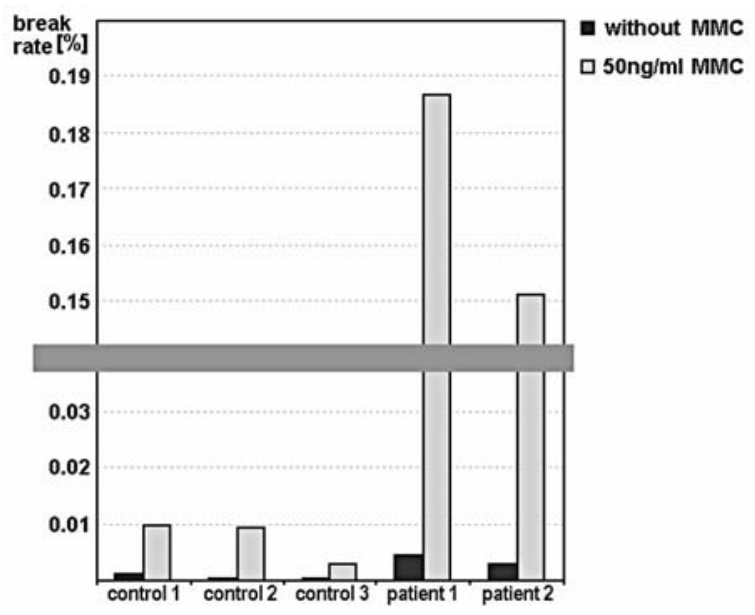

Figure 1. Spontaneous (dark gray columns) and with mytomycin C (MMC) induced break rates (light grey columns) per metaphase. FA patients 1 and 2 have significant higher break rates than the three normal controls.

It was shown previously, that MMC induced chromosomal breaks in metaphases of FA-lymphocytes co-localize on the cytogenetic level with FS (16-18). Based on these cytogenetic studies and on the findings that FS co-localize with evolutionary conserved and tumor associated break-points on the molecular-cytogenetic level we hypothesized a molecular co-localization of FS with FA-break-point regions. To address this question in more detail we present the first molecular cytogenetic investigation of FA-bp in comparison to FS.

\section{Materials and methods}

Materials. Patient 1 was a 16-year old female FA-patient with complementation group FANC-A (mutation: c.1567-1G $\rightarrow$ C). Patient 2 was a female, 19 years of age having a FA of subtype FANC-C (mutation: 1BP del322delG). Stimulated peripheral blood lymphocytes were used from patient 1 , while from patient 2 an immortalized B-lymphoblastoid cell line (ECACC: GM12794) was available. Clinically healthy females aged 24, 26 and 35-years served as negative controls.

Cytogenetics. For patient 1 and the three controls $5 \mathrm{ml}$ peripheral venous blood was cultivated according to standard procedures (19). Chromosomal breaks were induced by adding MMC in final concentration of $50 \mathrm{ng} / \mathrm{ml} 24 \mathrm{~h}$ after culture set up and $48 \mathrm{~h}$ before harvesting in one out of two culture flasks. The second culture not treated with MMC served as negative control. Standard protocols were used for preparation of fixed cell suspension. The lymphoblastoid cell line of patient 2 was treated the same way, excluding growth stimulation by phytohemagglutinine.

Molecular cytogenetics. Thirty-four BAC clones were obtained from the Children's Hospital Oakland Research Institute (CHORI), Oakland, CA, USA or kindly provided by the Sanger Center. A list of all bacterial artificial chromosome (BAC) clones is given in Table I. BAC DNA was isolated, PCR-amplified and labeled as described (20). Single and multicolor fluorescence in situ hybridization (FISH) techniques were performed according to published protocols (20).
Cytogentic and molecular cytogenetic analysis were done with an Axioplan 2 fluorescence microscope (Zeiss, Jena, Germany), a standard CCD-camera and the software ISIS (MetaSystems, Altlussheim, Germany). Chromosome banding was achieved by DAPI counterstaining (4',6-diaminidino-2phenylindoleb, Sigma).

Only chromosome (22\%) or chromatide breaks $(78 \%)$ were included in the break-point analysis (Fig. 7). Reunion figures (inter and intra chromatide exchanges caused by two or more chromatide lesions and subsequent rearrangement of the chromatides) were taken into consideration only for the absolute number of breaks to determine the breakage rate. For every chromosome involved in a reunion figure one chromosome break was counted. Every complex karyotype (metaphase with a highly aberrant karyotype) was recorded with 12 breaks in general.

Statistical analysis. For the calculation of the standard deviation the following formulae according to Brenstein et al (21) were used:

$$
\begin{aligned}
\bar{x} & =\frac{1}{n} \sum_{i=1}^{n} x_{i} \\
v_{i} & =\bar{x}-x_{i} \\
\tilde{\delta}_{A M} & =\sqrt{\frac{\sum_{i=1}^{n} v_{i}^{2}}{n(n-1)}}
\end{aligned}
$$

\section{Results}

Breakage rates. An initial cytogenetic analysis of the studied FA patients and three healthy controls revealed the expected enhanced breakage rate in FA-patients when cultures were treated with MMC as well as without induction (Fig. 1). In control persons spontaneous breakage rates deviate from $0.0004( \pm 0.004)$ to $0.0012( \pm 0.009)$ (breaks per chromosome number; average of all metaphases; $n=54-56$ metaphase spreads), whereas FA-patients ranged from $0.0028( \pm 0.0008)$ to $0.0044( \pm 0.0014)(n=105-106$ metaphase spreads $)$. Cells of control persons induced with MMC reached between 0.0028 $( \pm 0.001)$ and $0.01( \pm 0.003)(n=49-56$ metaphase spreads $)$ and FA-patients between $0.1512( \pm 0.0092)$ and $0.1870( \pm 0.0093)$ $(n=186-240$ metaphase spreads).

Cytogenetic co-localization and frequency. A more detailed examination of DAPI-banded chromosomes revealed that $50.58 \%$ of all FA-bp are located within FS regions on a cytogenetic level (Fig. 2). FS that normally appear frequently in healthy individuals after induction, for example FRA3B and FRA16D, were quite rare, and those ones normally rare were observed often in FA-chromosomes (see Table II).

Molecular cytogenetic co-localization. FA-bp located within an FS-region on the cytogenetic level were further analyzed by FISH-technique in order to reveal or exclude a colocalization also on the molecular-cytogenetic level. Twentynine FS were analyzed by 38 different BAC-probes (Table I) characterizing these FS. BAC-probes were selected 


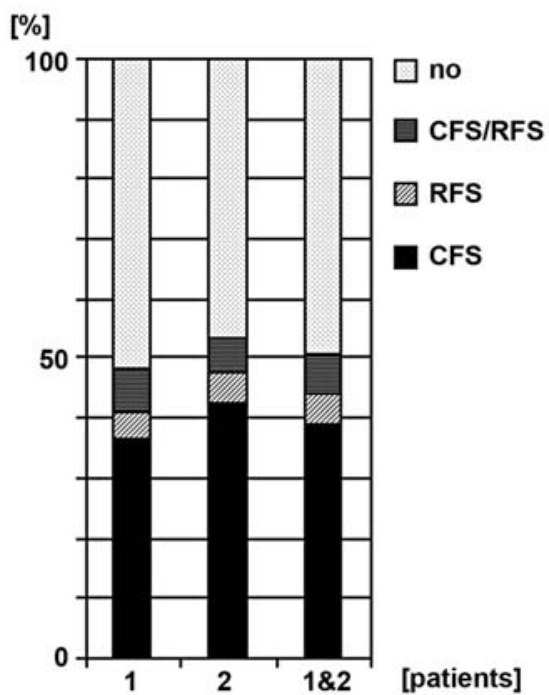

Figure 2. FA-bp show on the cytogenetic level a concordance of $50.58 \%$ with FS. CFS, common fragile sites; no, no concordance; RFS, rare fragile sites.

Table II. Frequencies of selected FS in normal controls compared to FA-patients. ${ }^{\text {a }}$

\begin{tabular}{ccc}
\hline FS/FA-bp & $\begin{array}{c}\text { Frequency in normal controls } \\
\text { after FS-induction with }\end{array}$ & $\begin{array}{c}\text { Frequency of } \\
\text { aphidicolin }(\%)\end{array}$ \\
& FA-bp $(\%)$
\end{tabular}

\begin{tabular}{lrl}
\hline FRA3B & 14.15 & 1.0 \\
FRA16D & 7.58 & 1.0 \\
FRA1E/M & 0.07 & 5.0 \\
FRA1D & 0.01 & 4.0 \\
FRA1J & 0.12 & 3.0 \\
FRA1A & 0.49 & 3.0 \\
FRA4C & 2.18 & 3.0 \\
\hline
\end{tabular}

aFRA1A, FRA1D, FRA1E and FRA1J are more frequently observed as FA-bp while it is vice versa for FRA3B, FRA4C and FRA16D.

according to molecular genetic and molecular cytogenetic data from the literature (Table I). FS-specific FISH proofed for the first time a molecular cytogentic co-localization of FS and FA-bp in 24 out of 29 analyzed FS (82.8\%), respectively. Fig. 3 shows two examples for FA-bp mapping by FSspecific BACs. For each FA-bp association with an FS 2-3 or 4 or more metaphases with the corresponding FA-bp were analyzed (Fig. 4).

Break rate and band level/GTG-light bands. Additionally, this study demonstrated that the observable break rate in FA or control lymphocyte preparations correlates positively with the chromosomal band level. At a level of 450 bands per haploid karyotype the break rate was on average 0.1419 $( \pm 0.008$; patients 1 and 2$)$, whereas it was $0.2785( \pm 0.0148$; patient 1 and 2) at a 650-band level (Fig. 5). Furthermore, it is remarkable that $58 \%$ of all breaks observed (patients 1 and 2,

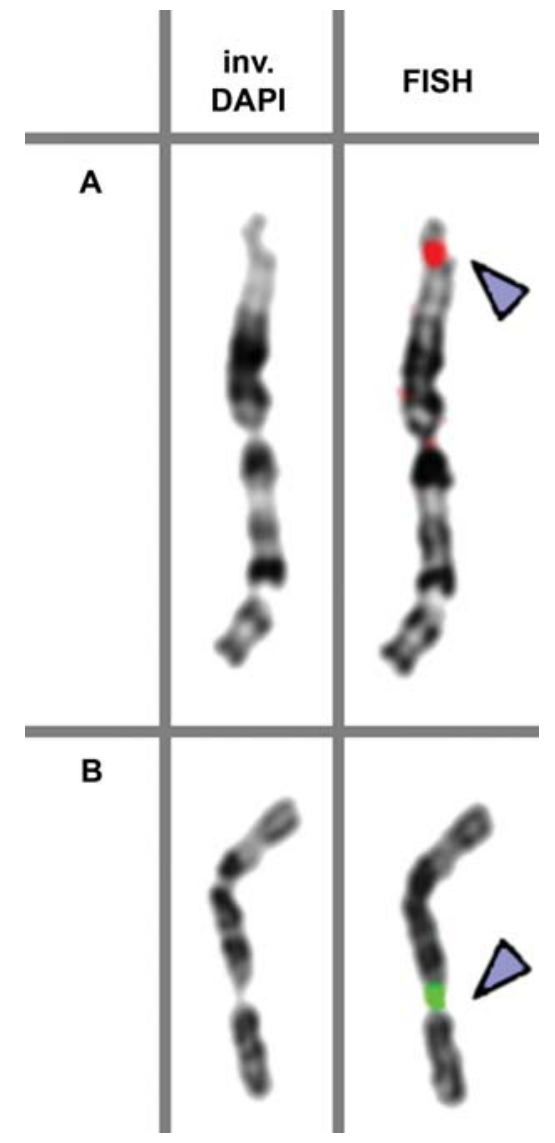

Figure 3. Two examples for the molecular cytogenetic co-localization of an FS and a FA-bp are depicted. inv DAPI, inverted DAPI-staining; FISH, result of FISH using FS-specific BAC-probes. (A) A chromosome 1 with a FA-associated break in 1p36 was hybridized with the BAC-probe RP1119M4 specific for the FS FRA1A. The observed red signal is located within the FA-bp (red arrow). (B) A chromosome 6 with a FA-associated break in 6q21 hybridized with the FRA6F-specific BAC RP11-188A15 revealed a green signal directly within the break (red arrow).

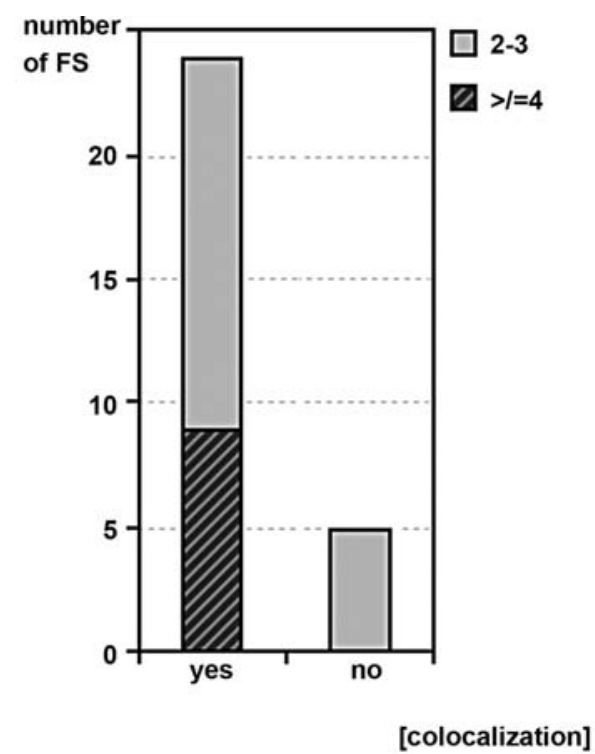

Figure 4. Twenty-four out of 29 different analyzed FA-bp showed a colocalization with an FS. The dark gray marked parts of the first column represent FA-bp which could be studied by FISH between 4 up to 13 times $(\geq 4 x)$. The remaining FA-bp were found between 2 and 3 (2-3) times only; however, in 15 of these cases the results showed co-localization of FA-bp and FS-specific BACs. In 5 cases no co-localization could be detected (see also Table I). 


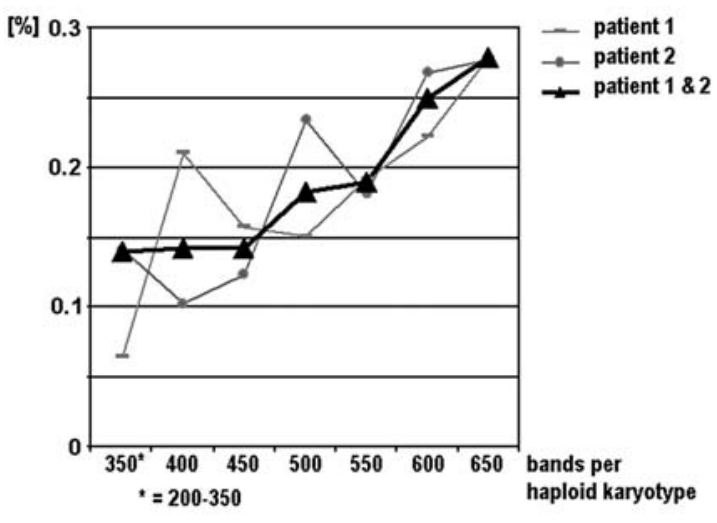

Figure 5. A positive correlation between chromosomal haploid band level and FA-bp per metaphase and FA patients 1 and 2 was observed.

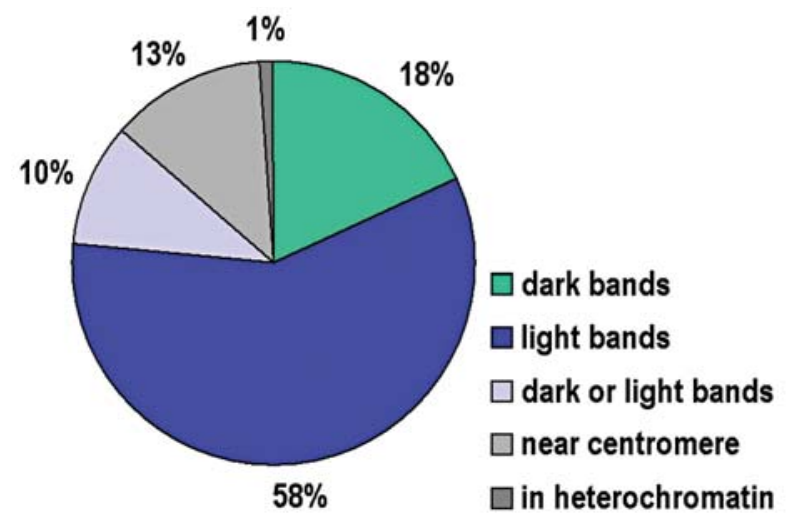

Figure 6. Fifty-eight percent of all cytogenetic breaks are localized in GTGlight bands, and $18 \%$ in dark bands. In $10 \%$ the break was located on the boarder of a dark and light band.

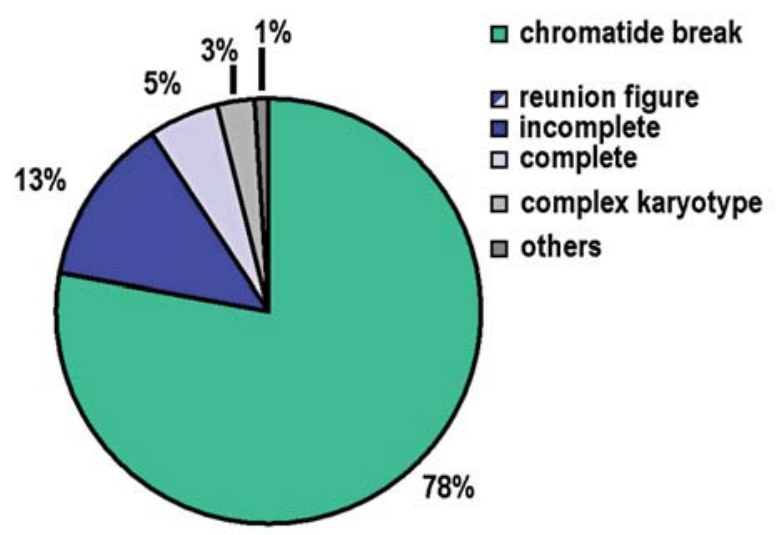

Figure 7. Seventy-eight percent of all break-events were chromatide breaks. Metaphases with 12 or more aberrations were described as complex karyotype.

400-450 band-level, $\mathrm{n}=263$ breaks) occurred in GTG-light bands and only $18 \%$ in GTG-dark bands (Fig. 5). The remaining $24 \%$ of breaks were located in bands next to the centromere $(13 \%)$, in heterochromoatic regions of the chromosomes $(1 \%)$ or between GTG light and dark bands (10\%).

\section{Discussion}

Cytogenetic co-localization and frequency. About $50 \%$ of all breaks analyzed for their cytogenetic localization in FA patients were located within FS (Fig. 2). Thus, FS are obviously overrepresented within the FA-bp. At present about 120 FS are reported in the literature (22). Overall this finding implies an important role of FS in FA associated chromosome fragility.

How can the expression of FS in FA-metaphase chromosomes be explained? i) FA on the one side and FS on the other share parts of their molecular pathways. Previous studies were able to show that several proteins involved in FA pathway also play a role in FS instability. Casper and colleagues (8) demonstrated that the loss of ATR, a protein interacting with the FA pathway, leads to an elevated FS expression. Arlt and colleagues (23) provided evidence that BRCA1, which builds nuclear foci with FANC-D2, -J, D1 etc. in case of DNA-damage, is essential for FS-stability. When down-regulated, FS expression is two- to four-fold compared to levels in control cells with normal BRCA1 expression. Another study by Howlett and coworkers illustrated that a knock-out of the FA-pathway leads to a higher expression of APH induced FS (24). These findings can possibly explain why the FS expression in the two analyzed FA-patients is very high.

ii) The concordance of FS and FA break-points can be explained by mechanistic reasons: the chromosomal band level, i.e., the decondensation of the chromosome (25) influences the break rate observed in chromosomes (Fig. 5). This positive correlation between band level and break rate can be explained by a mechanistic hypothesis: a high banding level seems to boost chromatide breaks for mechanic reasons, as it seems to be conceivable that long chromosomes with more decondensed chromatin structure are more affected by discontinuities during the process of chromosome preparation. Here we postulate that this plays a role in all chromosomes, also in chromosomes of healthy individuals. It is also possible that the break rates in different band levels are the same, but can only be seen when they are elongated to a certain extent, as is the case in higher band levels. The observation that light euchromatic bands are more often subject to breaks than dark heterochromatic bands (Fig. 6) also indicates that chromosome structure seems to play an important role in chromosome fragility such as the band level: areas with decondensed chromatin structure (light euchromatic bands) are as locus minoris resistentiae predisposed for expressing breaks first.

iii) The high FS expression in FA cells can be caused by a similar effect of APH and MMC. APH is an inhibitor of the DNA-polymerase $\alpha$ and $\delta(6,26)$ whereas MMC is an alkylating substance. Both inhibit the replication of DNA, but it has to be taken into consideration that FA-chromosomes also show a higher incidence of breaks and gaps even without MMC, so that the defect of the DNA-repair seems to be crucial for FS-expression.

In conclusion, it was shown that FS do play a dominant role in FA. This first molecular cytogenetic study verified a co-localization of FS and FA-bp in two different FA complementation groups. 
As FS are considered important factors in terms of developing cancer, it is reasonable to characterize more of the so far cytogenetically described FS on the molecular or molecular cytogenetic level. Our observation that FS, which normally occur infrequently in aphidicolin induced cells, can be found frequently in FA-chromosome spreads makes FA a model system for the fine-mapping of infrequent FS. The link between chromosomal instability and cancer is an intriguing one and needs further investigations especially as far as chromosome structure is concerned.

\section{Acknowledgments}

This study was supported in parts by Evangelische Studienwerk e.V. Villigst, IZKF Jena (Promotionsstipendium to C.S. and K.W., and Start-up S16 to AW), IZKF together with the TMWFK (TP 3.7 and B307-04004), Ernst-Abbe-Stiftung, DFG (LI 820/15-1) and Deutsche Fanconi-Anämie-Hilfe e.V..

\section{References}

1. Taniguchi T: Fanconi Anemia [Fanconi Pancytopenia] Available at: http://www.ncbi.nlm.nih.gov/bookshelf/br.fcgi?book=gene\& part=fa Accessed on June 19, 2009.

2. Sasaki MS and Tonomura A: High susceptibility of Fanconis anemia to chromosome breakage by DNA crosslinking agents. Cancer Res 33: 1829-1836, 1973.

3. Kalb R, Neveling K, Herterich S and Schindler D: Fanconi Anemia. A paradigmatic disease for the understanding of cancer and aging. Kap Fanconi Anemia Genes: Structure, mutations, and genotype-phenotype correlations. Karger, Basel, pp39-55, 2007.

4. Patel KJ: Fanconi anemia and breast cancer susceptibility. Nat Genet 39:142-143, 2007.

5. Le Beau MM, Rassool FV, Neilly ME, Espinosa R III, Glover TW, Smith DI and McKeithan TW: Replication of a common fragile site, FRA3B, occurs late in S phase and is delayed further upon induction: implications for the mechanism of fragile site induction. Hum Mol Genet 7: 755-761, 1998

6. Ikegami S, Taguchi T, Ohashi M, Oguro M, Nagano H and Mano Y: Aphidicolin prevents mitotic cell division by interfering with the activity of DNA polymerase-alpha. Nature 275: 458-460, 1978.

7. Zhang $\mathrm{H}$ and Freudenreich $\mathrm{CH}$ : An AT-rich sequence in human common fragile site FRA16D causes fork stalling and chromosome breakage in S. cerevisiae. Mol Cell 27: 367-379, 2007.

8. Casper AM, Nghiem P, Arlt MF and Glover TW: ATR regulates fragile site stability. Cell 111: 779-789, 2002.

9. Hellmann A, Rahat A, Scherer SW, Darvasi A, Tsui LP and Kerem B: Replication delay along FRA7H, a common fragile site on human chromosome 7, leads to chromosomal instability. Mol Cell Biol 20: 4420-4427, 2000.

10. Mishmar D, Rahat A, Scherer SW, Nyakatura G, Hinzmann B, Kohwi Y, Mandel-Gutfroind Y, Lee JR, Drescher B, Sas DE, Margalit H, Platzer M, Weiss A, Tsui LC, Rosenthal A and Kerem B: Molecular characterization of a common fragile site (FRA7H) on human chromosome 7 by the cloning of a simian virus 40 integration site. Proc Natl Acad Sci USA 95: 8141-8146, 1998.
11. Glover TW, Hoge AW, Miller DE, Ascara-Wilke JE, Adam AN, Dagenais SL, Wilke CM, Dierick HA and Beer DG: The murine Fhit gene is highly similar to its human orthologue and maps to a common fragile site region. Cancer Res 58: 3409-3414, 1998.

12. Coquelle A, Pipiras E, Toledo F, Buttin G and Debatisse M: Expression of fragile sites triggers intrachromosomal mammalian gene amplification and sets boundaries to early amplicons. Cell 89: 215-225, 1997.

13. Sankoff D, Deneault M, Turbis P and Allen C: Chromosomal distributions of breakpoints in cancer, infertility, and evolution. Theor Popul Biol 61: 497-501, 2002.

14. Pichiorri F, Ishii $\mathrm{H}$, Okumura $\mathrm{H}$, Trapasso F, Wang $\mathrm{Y}$ and Huebner K: Molecular parameters of genome instability: roles of fragile genes at common fragile sites. J Cell Biochem 104: 1525-1533, 2008.

15. Manvelyan M, Schreyer I, Höls-Herpertz I, Köhler S, Niemann R, Hehr U, Belitz B, Bartels I, Götz J, Huhle D, Kossakiewicz M, Tittelbach H, Neubauer S, Polityko A, Mazauric ML, Wegner R, Stumm M, Küpferling P, Süss F, Kunze H, Weise A, Liehr T and Mrasek K: Forty-eight new cases with infertility due to balanced chromosomal rearrangements: detailed molecular cytogenetic analysis of the 90 involved breakpoints. Int J Mol Med 19: 855-864, 2007.

16. Schroeder TM, Anschutz F and Kopp A: Spontaneous chromosomal aberrations in family panmyelopathy. Humangenetik 1: 194-196, 1964.

17. Bloom GE, Warner S, Gerald PS and Diamond LK: Chromosome abnormalities in constitutional aplastic anemia. N Engl J Med 274: 8-14, 1966.

18. Koskull HV and Aula P: Nonrandom distribution of chromosome breaks in Fanconis anemia. Cytogenet Cell Genet 12: 423-434, 1973.

19. Verma RS and Babu A: Human Chromosomes, Principles and Techniques. 2nd edition. McGraw-Hill, Inc., New York, St. Louis, Toronto, 1995.

20. Liehr T, Weise A, Heller A, Starke H, Mrasek K, Kuechler A, Weier HU and Claussen U: Multicolor chromosome banding (MCB) with YAC/BAC-based probes and region-specific microdissection DNA libraries. Cytogenet Genome Res 97: 43-50, 2002.

21. Brenstein IN, Semendjajew KA, Musiol G and Mühlig H: Taschenbuch der Mathematik. Thun, Frankfurt am Main, Harri Deutsch, 1997.

22. Lukusa T and Fryns JP: Human chromosome fragility. Biochim Biophys Acta 1779: 3-16, 2008.

23. Arlt MF, Xu B, Durkin SG, Casper AM, Kastan MB and Glover TW: BRCA1 is required for common-fragile-site stability via its G82)/M checkpoint function. Mol Cell Biol 24: 6701-6709, 2004.

24. Howlett NG, Taniguchi T, Durkin SG, D'Andrea AD and Glover TW: The Fanconi Anemia pathway is required for the DNA replication stress response and for the regulation of common fragile site stability. Hum Mol Genet 14: 693-701, 2005.

25. Claussen U, Michel S, Mühlig P, Westermann M, Grummt UW, Kromeyer-Hauschild K and Liehr T: Demystifying chromosome preparation and the implications for the concept of chromosome condensation during mitosis. Cytogenet Genome Res 98: 136-146, 2002.

26. Glover TW, Berger C, Coyle J and Echo B: DNA polymerasealpha inhibition by aphidicholin induced gaps and breaks at common fragile sites in human chromosomes. Hum Genet 67: $136-142,1984$. 